\title{
Conhecimento matemático especializado de professores da educação infantil e anos iniciais: conexões em medidas
}

Rosa Di Bernardo*

Milena Soldá Policastro** Alessandra Rodrigues de Almeida***

Miguel Ribeiro****

*(Universidade Estadual de Juscier Mamoré de Melo Campinas - Unicamp, Campinas, São Mariana Aiub******

Paulo, Brasil)

**(Universidade Estadual de Campinas - Unicamp, Campinas, São Paulo, Brasil)

***(Pontifícia Universidade Católica de Campinas, PUC Campinas, São Paulo, Brasil)

$\star \star \star \star$ (Universidade Estadual de Campinas - Unicamp, Campinas, São Paulo, Brasil)

$\star \star \star * *$ (Universidade Estadual de Campinas - Unicamp, Campinas, São Paulo, Brasil)

$\star \star \star \star \star \star *$ (Universidade Estadual de Campinas - Unicamp, Campinas, São Paulo, Brasil)
Resumo: Neste trabalho buscaram-se evidências do conhecimento especializado (na perspectiva do Mathematics Teachers' Specialized Knowledge) dos professores da educação infantil e dos anos iniciais do ensino fundamental, ao discutirem uma tarefa conceitualizada para desenvolver o conhecimento do modo como as noções de quantidade, unidade e grandeza se articulam. Os resultados revelaram que os participantes associam medida ao estabelecimento de uma referência de comparação, seguida da indicação de um valor numérico e um símbolo para representação/ indicação da quantidade e do que foi medido. As conexões entre as noções de quantidade, unidade e grandeza permitem detalhar as implicações da opção por cada um dos eixos de conexão e, em termos de formação, abrem-se possibilidades investigativas ao assumir cada um dos eixos como foco.

Palavras-chave: Conhecimento especializado. Medidas. Formação de professores. Anos iniciais. 
INTRODUÇÃO

$\mathrm{O}$ ato de medir está presente no cotidiano das pessoas, que, desde a infância, são colocadas em situações em que precisam efetivamente realizar medidas ou, então, utilizar raciocínios correlatos às medidas. De práticas comuns, como a ida ao supermercado, a contextos mais particulares, como as pesquisas em laboratórios, as medidas têm papel fundamental no modo como as sociedades se organizaram desde a pré-história. Em particular, podemos considerar que "toda gente, nas mais variadas circunstâncias, qualquer que seja sua profissão, tem a necessidade de medir" (CARAÇA, 1963, p. 29) e, portanto, o tema das medidas desempenha um papel importante nos currículos internacionais desde a educação infantil (ITÁLIA, 2012; NORWEGIAN, 2017; PORTUGAL, 2013).

Também nos documentos oficiais brasileiros, que orientam a organização dos currículos como conteúdos a serem trabalhados com os alunos desde a educação infantil (BRASIL, 1997, 2018), o eixo temático denominado Grandezas e Medidas encontra-se presente. Um foco no ensino e aprendizagem da medida é considerado essencial (NATIONAL GOVERNORS ASSOCIATION CENTER FOR BEST PRACTICES; COUNCIL OF CHIEF STATE SCHOOL OFFICERS, 2010), pois este é um dos temas nucleares para que os alunos possam acessar o mercado de trabalho e as áreas das ciências, tecnologias, matemática e engenharias, as quais, incorporadas no cluster da Stem ${ }^{1}$, são consideradas como as mais promissoras para o desenvolvimento social (RIBEIRO et al., 2016).

Embora os documentos nacionais destaquem a forte relevância social do caráter prático e utilitário das medidas (BRASIL, 1997, 2018), os alunos apresentam baixos níveis de aprendizagem nas tarefas relacionadas a grandezas e medidas (BERTOLINO, 2017). A essas dificuldades com esse tema, podemos juntar aquelas reveladas pelo professor (POLICASTRO; ALMEIDA; RIBEIRO, 2018).

Quando se trata do tópico de grandezas e medidas, é natural vincular essa discussão às noções ao redor do sentido de número. Este, quando não é bem consolidado, pode implicar dificuldades nas aprendizagens matemáticas dos alunos. Em contrapartida, os alunos nos quais o sentido de número é desenvolvido tendem a possuir maior desenvoltura em relação à matemática

A Science, Technology, Engineering e Mathematics (Stem) Learning é a maior fornecedora de suporte de educação e de carreiras em ciência, tecnologia, engenharia e matemática, e é composta por uma parceria entre governo, organizações de caridade e empresários, com foco no desenvolvimento de conhecimentos relacionados com as disciplinas Stem. 
(MUIR, 2012). Portanto, é preciso que os aprendizes sejam colocados em atividades diárias nas quais se proporcionem múltiplas discussões sobre o sentido de número, os seus usos e as ideias associadas a tais sentidos. Dessa forma, articular as noções do sentido de número ao trabalho com o tópico de grandezas e medidas deverá contribuir significativamente para as aprendizagens discentes.

A pesquisa em educação matemática mostra que, dentre os fatores que se podem controlar, o que maior impacto possui nas aprendizagens dos alunos é o conhecimento do professor (BALL; HILL; BASS, 2005; GROSSMAN, 2010; HILL; ROWAN; BALL, 2005; NYE; KONSTANTOPOULOS; HEDGES, 2004). Porém, para que tal impacto tenha efeito, esse conhecimento não pode ser considerado de forma ampla - e do mesmo tipo para professores de áreas distintas -, mas é preciso ter em conta as especificidades da atuação do professor em cada uma das áreas de conhecimento (RIBEIRO, 2018), o que leva a que esse conhecimento seja especializado. Essa especialização é considerada no domínio tanto do conhecimento matemático como do conhecimento pedagógico do conteúdo matemático e, a esse respeito, considera-se a conceitualização do Mathematics Teachers' Specialized Knowledge - MTSK (CARRILLO et al., 2013).

De forma a contribuir para a melhoria da prática e da formação, tornase essencial que a formação centre-se onde é efetivamente necessária (RIBEIRO; CARRILLO, 2011). Para essa melhoria da prática, é essencial um foco no conhecimento especializado do professor e, dentre os temas matemáticos, um dos que se configuram como problemáticos é o tema da medida (HEUVEL-PANHUIZEN; ELIA, 2011; POLICASTRO; ALMEIDA; RIBEIRO, 2018; SILVA; BELLEMAIN; BORBA, 2016). Com essa problemática em mãos, e buscando contribuir para a discussão que permita ampliar o entendimento do conhecimento do professor no âmbito da medida, buscamos respostas para a seguinte questão: Que dimensões do conhecimento especializado do professor que ensina matemática revelam professores em exercício sobre o modo como se relacionam as noções de unidade, grandeza e quantidade, $e$ como essas relações sustentam o conhecimento da atividade de medir? 
Na Base Nacional Comum Curricular - BNCC (BRASIL, 2018), as medidas são consideradas desde a educação infantil - associadas explicitamente ao campo experiencial Espaços, Tempos, Quantidades, Relações e Transformações - até os anos finais, associadas à unidade temática Grandezas e Medidas. 0 desenvolvimento de atividades de medição desde a educação infantil possibilita a criação de um vínculo direto entre a matemática e o cotidiano do aluno, e propicia que os temas matemáticos estudados tornem-se mais concretos e significativos, contribuindo para dar significado ao mundo que nos rodeia (SILVA et al., 2016).

Desde a educação infantil, é essencial que a escola, e necessariamente a prática do professor, possibilite oportunidades de aprendizagem (ALSINA, 2017; HIEBERT; GROUWS, 2007; WATTS et al., 2018) para que as crianças possam desenvolver, desde cedo, conhecimentos, habilidades e competências matemáticas. Entre os elementos centrais desse conhecimento, incluem-se o sentido de número e de operação, de noções geométricas “elementares” e do significado e processo de medição. Um conhecimento associado ao sentido da medição corresponde a deter um conjunto de formas de fazer e entender a medida de modos múltiplos - padronizadas ou não padronizadas (RIBEIRO et al., 2017) -, que permitam ao indivíduo efetuar medições de maneira fácil e apropriada. 0 desenvolvimento do sentido de medição implica um processo complexo, que tem como elementos centrais a percepção e a comparação de quantidades mensuráveis e é integrado com o uso de técnicas de medição e estratégias de estimativa em situações contextualizadas e significativas (para os alunos).

Segundo Clements e Stephan (2004), medir é um processo que associa uma sequência de procedimentos (e raciocínios envolvidos a esses procedimentos) à atribuição de um número a quantidades contínuas. 0 processo cognitivo das crianças sobre as medidas de comprimento, por exemplo, ocorre primeiro pela associação da palavra à quantidade que esta representa. Em seguida, realizam a comparação de dois objetos, evidenciando suas semelhanças e diferenças (mais longo/mais curto; mais leve/mais pesado), para, posteriormente, associar um número à quantidade e verbalizar (CLEMENTS; STEPHAN, 2004). Para realizar uma medição, é necessário estabelecer um padrão único de comparação para todas as grandezas de mesma espécie, ou seja, uma unidade de medida. Além disso, é essencial poder verificar quantas vezes a unidade de medida cabe na grandeza que se mede, atribuindo um valor numérico ao resultado dessa medição (CARAÇA, 1963). 
Clements e Stephan (2004) consideram seis princípios associados à atividade de medir unidimensionalmente (comprimento): (a) partição - relaciona-se com a atividade mental de dividir o objeto em unidades menores e de mesmo comprimento -, considerando aqui o caso particular de a unidade ser menor que o objeto que se vai medir; (b) unidade de iteração - corresponde à habilidade de pensar em um comprimento como referência para se deslocar em todo o comprimento do objeto, de modo a que ambas as extremidades (final e inicial) desse comprimento de referência coincidam no processo de iteração, o que implica não deixar espaços entre duas unidades subsequentes, nem sobrepor unidades adjacentes; (c) transitividade - corresponde ao processo de, por meio de estimativa ou dedução, obter uma relação de igualdade ou desigualdade (superior ou inferior) de uma grandeza e estendê-la a outros dois ou mais objetos; (d) conservação - associa-se à compreensão de que qualquer movimento (translação ou rotação) no objeto que será/foi medido manterá os comprimentos; (e) acumulação da distância - corresponde ao entendimento de que, no processo de iteração de uma unidade de comprimento ao longo do comprimento do objeto que se mede, realiza-se a contagem da quantidade de iterações, e o valor numérico (número de vezes que se repetiu a unidade) corresponde ao espaço coberto por essas unidades contadas até aquele ponto; (f) relação da medida com um valor numérico - associa-se à reorganização da compreensão, pelo indivíduo, do processo de contagem de quantidades discretas para quantidades contínuas. Embora esses princípios tenham sido elencados especificamente associados à medição de comprimento, todos podem ser transpostos para uma medida não necessariamente relacionada ao comprimento (HEUVEL-PANHUIZEN; ELIA, 2011), considerando-se as adaptações convenientes. Para que os alunos possam ter oportunidades de aprendizagem para desenvolver um conhecimento de cada um desses princípios, articulando-os na ação de medir, torna-se essencial que o professor detenha um conhecimento matemático sobre esses princípios e sobre formas de potencializar as discussões matemáticas com os alunos.

Ao mesmo tempo, quando se trata do tópico de grandezas e medidas, não é possível desvincular essa discussão de uma das noções matemáticas que subjazem aos fundamentos da atividade de medir: a noção de número. 0 número pode ser compreendido a partir de três sentidos: cardinal, ordinal e nominal (CEBOLA, 2002) e, no caso das grandezas e medidas, a noção de cardinalidade (quantificação) compõe uma das etapas no processo associado à atividade de medir, isto é, relaciona-se com a atribuição de um valor numérico à medida. Em última instância, quando associado a um símbolo que representa a grandeza que se está medindo - o que não acontece, por 
exemplo, no caso dos ordinais, em que o símbolo “o”" ou “a”" não representa uma grandeza a ser medida, mas sim uma posição -, o valor numérico relacionado com essa medida sustenta a atribuição de significado da relação entre unidade de referência e grandeza.

O desenvolvimento do sentido de número e, em particular, do senso de magnitude relativa e absoluta dos números deverá contribuir, no contexto das medidas, para que os alunos possam reconhecer, diferenciar e relacionar quantidade, unidade de referência e grandeza, de forma a, efetivamente, compreender as conexões entre a quantidade que o número representa e a quantidade de unidades/partes da unidade. Isso permitirá que eles possam comparar essas quantidades, atribuindo sentido aos princípios associados à atividade de medir (MUIR, 2012).

O conhecimento do professor "desde sempre" tem sido foco de discussão, mas, nos últimos 30 anos, tendo como precursores os estudos de Shulman (1986), tem assumido um lugar de destaque na pesquisa em educação. Essas ideias de Shulman, sendo inovadoras para a época, se relacionam à educação geral e não consideram as especificidades do conhecimento do professor no âmbito de cada um dos temas/conteúdos que terão de abordar (BALL; THAMES; PHELPS, 2008; CARRILLO et al., 2013; RIBEIRO, 2018). No Brasil, um metaestudo recente (FIORENTINI; PASSOS; LIMA, 2016), sobre as pesquisas voltadas para o professor que ensina matemática, defendidas entre 2001 e 2012 (875 dissertações e teses), mostra que a grande maioria das pesquisas que focam o professor que ensina matemática e discutem, de alguma forma, o seu conhecimento, associa a prática apenas como um contexto em que a pesquisa decorre e as informações são coletadas; e utiliza como referentes teóricos, essencialmente, Shulman (1986) e Tardif (2002). Esses focos de atenção (nas generalidades), objetivando a compreensão por parte dos alunos, deixam à margem as muitas especificidades do conhecimento do professor de/que ensina matemática, e os resultados dessas pesquisas são válidos para qualquer outro contexto educativo que não o de ensino e aprendizagem da matemática.

Assim, de forma a contribuir para a melhoria da formação e da prática (um dos objetivos associados à realização da pesquisa que deu origem a este texto) ${ }^{2}$, torna-se essencial não deixar à margem as especificidades associadas

Este é um dos objetivos explícitos da pesquisa e formação que vem sendo realizada pelo grupo ClEspMat (grupo de pesquisa e formação Conhecimento Interpretativo e Especializado do Professor de/que Ensina Matemática) de que os autores fazem parte. 
ao processo de ensino e aprendizagem da matemática. É preciso, inclusive, considerar essas especificidades como um dos focos centrais das pesquisas (e necessariamente da formação).

Essencialmente em nível internacional, etendo como ponto de partida o trabalho precursor de Shulman (1986), têm emergido algumas conceitualizações do conhecimento do professor que assumem a importância e as especificidades do conhecimento matemático para a atuação do professor de/que ensina matemática. Algumas delas, que têm assumido maior destaque, referem-se ao Knowledge Quartet (ROWLAND et al., 2009); Mathematical Knowledge for Teaching (BALL; THAMES; PHELPS, 2008) e Mathematics Teachers' Specialized Knowledge (CARRILLO et al., 2013). Dentre estas, no contexto de nossa investigação, assumimos a conceitualização do Mathematics Teachers' Specialized Knowledge - MTSK (CARRILLO et al., 2013), também pelo fato de que ela considera como especializado todo o conhecimento do professor de/que ensina matemática - tanto o conhecimento matemático quanto o conhecimento pedagógico -, algo que é uma premissa e uma necessidade tida como essencial para a melhoria da formação a facultar, a partir dos resultados da pesquisa.

Nesse sentido, e dado que o conhecimento do professor assume um papel central nas aprendizagens e nos resultados dos alunos (BALL; HILL; BASS, 2005), torna-se essencial um aprofundamento no entendimento sobre os conteúdos e as dimensões desse conhecimento e das suas especificidades. Propor novas bases para a formação (inicial e continuada) do professor de/que ensina matemática e, necessariamente, (re)formular maneiras de contribuir para a melhoria de sua prática, centrando-se onde tal formação é efetivamente necessária (RIBEIRO; CARRILLO, 2011; RIBEIRO, 2018), requer um redirecionamento de foco. Tal redirecionamento implica não se deter exclusivamente nos aspectos transversais à formação humana ou em dimensões comuns a todos os professores - aquilo que alguns consideram didática geral -, pois, embora sejam necessariamente importantes, tais aspectos não correspondem às dimensões mais críticas da formação do professor.

O modelo teórico-analítico do MTSK é constituído por dois domínios (Mathematical Knowledge [MK] e Pedagogical Content Knowledge [PCK]), considerando, em cada um, três subdomínios; e, no centro, incluem-se as crenças do professor sobre a matemática e sobre o ensino e aprendizagem da matemática. 


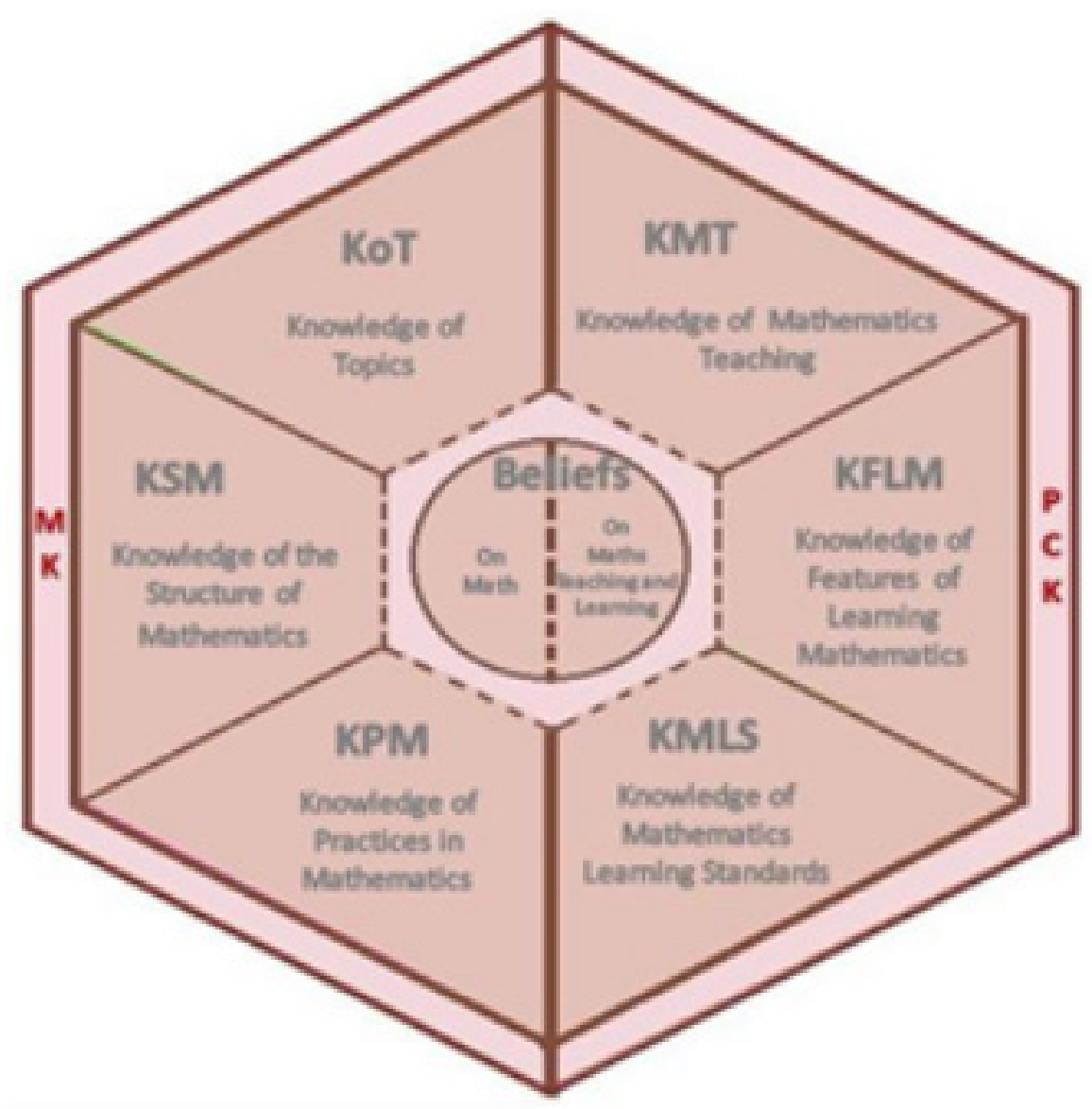

Fonte: Carrillo et al. (2013, p. 2989).

Considerar estes subdomínios do conhecimento do professor não corresponde a dizer que tal conhecimento está compartimentado, pois a forma como ele é encarado permite, por um lado, desenvolver uma abordagem teórica para modelar o conhecimento profissional do professor de/que ensina matemática (CARRILLO et al., 2013). Por outro lado, essa distribuição é considerada como uma ferramenta metodológica e analítica para investigar as distintas práticas do professor de/que ensina matemática (FLORES-MEDRANO et al., 2013), ao trabalhar com distintos tópicos.

Pelo contexto e pelo foco de trabalho que assumimos aqui, iremos voltar nossa atenção apenas aos subdomínios do conhecimento matemático do professor. A especialização do conhecimento do professor apoia-se na noção de que o conhecimento matemático lhe possibilite sustentar suas opções pedagógicas, de modo a permitir que os alunos compreendam o que fazem, como devem fazer e por que o devem fazer a cada momento, perspectivando atribuir significado a esse fazer não apenas de maneira pontual, mas em momentos futuros. 
Este conhecimento matemático do professor (os três subdomínios do modelo - KoT, KSM, KPM) deve ser complementar - mais amplo e profundo àquele que se espera que os alunos desenvolvam. Essa complementaridade considera necessário ao professor um conhecimento que permita promover e entender as potencialidades de relacionar definições, fundamentos, procedimentos "padronizados" ou "não padronizados", bem como as distintas formas de representação (verbal ou não verbal) de cada um dos diferentes tópicos e conteúdos. Também se considera importante, no âmbito dessa complementaridade, que o professor conheça o papel dos símbolos e o uso da linguagem associada em determinados contextos; que possa estabelecer conexões entre conteúdos em um mesmo tópico matemático ou entre tópicos distintos e, mais além, relacionar algum conhecimento desenvolvido anteriormente em outro tópico para desenvolver o conhecimento matemático dos alunos num contexto específico.

Assim, considera-se como parte integrante do conhecimento matemático do professor um conhecimento sobre os conceitos que lhe permita entender o que se faz, como se faz, por que se faz de determinada forma, quais os diferentes tipos de registro de representação e as características de determinado resultado. Mas que possibilite, também, conhecer múltiplas definições equivalentes para um mesmo conceito (Knowledge of Topics KoT). Um dos conteúdos desse subdomínio, no contexto de medidas, referese à necessidade de associar uma quantidade ao processo de medir, isto é, quando se reconhece que, ao dizer que a medida corresponde a 30, é necessário identificar a unidade de referência.

Outra dimensão a ser considerada é o subdomínio associado à estrutura da matemática, que inclui um conhecimento matemático amplo e profundo sobre cada um dos temas, assumindo uma perspectiva da sua integração, distribuição/exibição ao longo dos anos de escolaridade, bem como suas relações com estruturas mais amplas, ou mesmo com outras estruturas consideradas auxiliares ao pensamento matemático. Tal conhecimento sustenta uma prática letiva na qual o professor pode trabalhar a matemática elementar de um ponto de vista superior e vice-versa (Knowledge of the Structure of Mathematics - KSM). No contexto da medida, tal dimensão se associa ao conhecimento do professor de que os números podem assumir diferentes significados, e um deles está diretamente relacionado com a ideia de equivalência. Por exemplo, quando se estabelece uma relação de igualdade entre uma moeda de 1 real e 20 moedas de 5 centavos, faz-se necessário reconhecer que os números envolvidos na equivalência, embora sejam diferentes, representam as mesmas quantidades. 
Ainda incluído no conhecimento matemático do professor, há também um conhecimento associado às formas de fazer matemática. Dentre essas, podem ser referidas as diferentes maneiras de demonstrar, os critérios a estabelecer para que uma generalização seja válida, o significado de definição ou o conhecimento da sintaxe matemática, incluindo também os conhecimentos de diferentes estratégias de resolução de problemas ou de modelagem (Knowledge of Practices in Mathematics - KPM). Em termos de medida, podese relacionar o conteúdo do conhecimento do professor, por exemplo, com o reconhecimento de que, para validar uma medida, é necessário relacionar noções de grandeza e de unidade.

\section{Contexto e método}

Esta investigação compõe uma parte de um projeto maior, que tem como foco o conhecimento interpretativo e especializado e as práticas matemáticas do professor que ensina matemática na educação infantil e nos anos iniciais. No presente trabalho, concentramo-nos no conhecimento especializado de professores que ensinam matemática, em particular no âmbito das medidas, revelado durante um processo de formação continuada, oferecido em formato de workshop, sob responsabilidade do grupo Conhecimento Interpretativo e Especializado do professor de/que ensina Matemática (CIEspMat), que vem se reunindo na Faculdade de Educação da Unicamp desde o início de 2018, no primeiro sábado de cada mês, buscando efetivar uma aproximação entre universidade, escola, pesquisa e prática, assumindo a formação como eixo aglutinador.

Esses encontros de formação têm a duração de quatro horas, e cada um deles trata de um tema matemático identificado na pesquisa como problemático para professores e alunos. O nosso contexto aqui corresponde a um dos workshops com foco nas medidas (este é um tema transversal abordado em conexão com outros temas, tais como a divisão), em que estiveram presentes sete participantes ${ }^{3}$ - professores da educação infantil (01), dos anos iniciais do ensino fundamental (03), dos anos finais do ensino fundamental (01), um estudante de Pedagogia e uma psicopedagoga.

3 Os nomes usados são fictícios. 
Um dos principais objetivos do workshop é o de aceder ao conhecimento especializado dos participantes no âmbito da medida e desenvolvê-lo. A dinamização da formação ${ }^{4}$ ocorre seguindo uma estrutura que permite aos professores experienciar práticas que, segundo a pesquisa, podem potencializar as discussões matemáticas em sala de aula com os alunos, com foco em uma tarefa - para a formação matemática do professor - de natureza e foco distintos das tarefas para os alunos (RIBEIRO, 2016). Por isso, as tarefas são conceitualizadas com objetivos didáticos (de formação) associados à possibilidade de que os professores implementem com os seus alunos uma parte da tarefa, isto é, que discutam (e desenvolvam) os aspectos do conhecimento matemático especializado associado ao tema trabalhado - em particular, requerido para a posterior implementação - com vistas a desenvolver a tarefa com os alunos; e que desenvolvam, nesse processo, 0 seu conhecimento para atribuir sentido e significado a produções de alunos de modo a lhes fornecer um feedback construtivo (RIBEIRO; MELLONE; JAKOBSEN, 2016). Das três partes da tarefa, iremos focar nossa atenção na primeira, associada a aceder ao conhecimento dos participantes relativamente ao que significa medir e desenvolvê-lo; a como se mede; ao que se mede; aos motivos pelos quais se mede; e a com o que medir.

Os participantes resolveram a tarefa em dois grupos (aqui o foco de atenção corresponde a um desses grupos), e as discussões em cada um foram gravadas em áudio e vídeo, assim como as discussões finais coletivas. Além disso, as produções escritas dos participantes foram coletadas. Em cada um dos grupos estava um pesquisador com o papel de incentivar as discussões - mas sem corrigir ou validar as respostas/comentários dos participantes - e de tomar notas relativamente aos aspectos do conhecimento do professor que se configuram como mais significativos para contribuir na busca de resposta à nossa questão de pesquisa. Essas anotações serviram como elementos desencadeadores para identificar episódios significativos nas gravações de áudio e vídeo que foram posteriormente transcritos.

Inicialmente, a análise dos episódios foi efetuada de forma independente, buscando identificar nas discussões evidências de MTSK dos participantes. A

\footnotetext{
As formações a que nos referimos são dinamizadas pelo grupo Conhecimento Interpretativo e Especializado do professor de/que ensina Matemática (CIEspMat), um grupo composto por doutores, doutorandos e mestrandos que têm como foco de trabalho (pesquisa e formação) o conhecimento e a prática do professor de/que ensina matemática, desde a educação infantil até o ensino superior (este último, com foco, também, nos formadores de professores).
} 
partir dessas evidências, buscaram-se relacionar os conteúdos do MTSK dos participantes com as três dimensões centrais desta investigação: as noções de quantidade, unidade e grandeza. Nessas relações, foi-se configurando a possibilidade de estabelecer conexões de tais noções (quantidade, unidade e grandeza) com a noção de comparar, ao mesmo tempo que emergiam as evidências de como o MTSK sustenta o conhecimento que os participantes revelam sobre a atividade de medir.

\section{ANÁLISE E DISCUSSÃO}

De um modo geral, os resultados indicam que os participantes revelam conhecimento sobre o tema da medida, associando medida essencialmente ao estabelecimento de uma referência para comparação, seguido da indicação de um valor numérico e de um símbolo que representa uma unidade (padronizada ou não padronizada) para indicação da grandeza.

Quando solicitados a refletir sobre "o que é medir", os professores associam as noções de unidade, grandeza e quantidade para atribuir sentido à noção que fundamenta a atividade de medir, que é a de comparação. Por outro lado, para responderem a esta questão de "o que é medir", os professores revelam conhecimentos associados a "como se mede" e "com o que se mede", o que denota alguns aspectos do conteúdo de conhecimento, quando confrontados com questões que não se associam ao desenvolvimento de uma prática pedagógica (em termos de que opções didáticas irão tomar). 0 espaço das respostas dos professores situa-se no âmbito do conhecimento pedagógico do conteúdo (PCK), embora a questão não se situe no espaço de como ensinar, mas contenha aspectos que sustentam essa prática - sempre e quando se pretende que os alunos possam entender o que fazem e por que o fazem.

\footnotetext{
A primeira coisa que me vem à cabeça é que medir tem a ver com comparar. Porque eu já sei que a gente tem as unidades de medida, então, quando você vai medir, você vai comparar com aquela unidade de medida determinada, você tem uma referência, então me vem a ideia que medir tem a ver com comparar, e aí depende o que você vai comparar: comparar o tamanho, comparar o volume, comparar a massa, comparar o tempo (Rúbia, professora dos anos iniciais do ensino fundamental)
}

A ideia intuitiva (e não necessariamente consciente) do que é medir associa-se à comparação. Assim, medir está relacionado com considerar uma unidade, uma grandeza e uma quantidade, com os procedimentos (como se mede) e aplicações, assumindo essas dimensões como os pilares que sustentam 
DI BERNARDO, Rosa; POLICASTRO, Milena Soldá; ALMEIDA, Alessandra Rodrigues de; RIBEIRO, Miguel;

a noção transversal de comparar (KoT - fundamentos, procedimentos e aplicação). Rúbia revela um conhecimento de que comparar é algo transversal às medidas que se abordam no contexto escolar - "tamanho", "volume", "massa", "tempo" (KSM, conexões transversais) -, ainda que, ao referir “tamanho”, se possa considerar uma multiplicidade de dimensões de medida, a princípio, três. Porém, é possível ampliar tais noções, associando “maior tamanho" a maior peso/massa/volume (KoT, o que medir), o que se vincula a um uso adequado da linguagem matemática (POLICASTRO; ALMEIDA; RIBEIRO, 2018).

Outra visão complementar associa (reduz/limita) a medida à determinação de uma quantidade - um foco na prática matemática como um produto final, e não como uma prática em que o percurso pode ser o mais significativo -, obtendo, obviamente, o resultado final correto.

Faz sentido. É para você ter a ideia da quantidade, né? (Amanda, psicopedagoga).

Isso que eu ia falar, o objetivo de você medir é você quantificar, seja lá o que for (Wilson, professor dos anos finais do ensino fundamental).

Assim, a prática de medir (que não é exclusivamente matemática - KSM) tem como objetivo último a quantificação (KPM - aplicabilidade da matemática), revelando também um conhecimento sobre os porquês associados a essa atividade de medir (“o objetivo de você medir é você quantificar" - KoT procedimentos e fundamentos), algo que, de fato, está também relacionado com um dos princípios que fundamentam os processos e os raciocínios vinculados à medição (CLEMENTS; STEPHAN, 2004).

A noção de medida está apoiada na ideia de número como quantidade (CEBOLA, 2002) e na noção de que é possível comparar essa quantidade com outra, considerada como referência (unidade de medida), associando a tal comparação um valor numérico (CLEMENTS; STEPHAN, 2004) para indicar a quantidade de vezes que a unidade está contida no todo que se está medindo.

Inclusive, a gente trabalha com eles..., os próprios números eles têm funções diferentes, você encontra números no dia a dia que são usados para representar coisas diferentes: tem o número de contar, de representar uma quantidade, o cardinal, no caso; tem o número para medir: quando você põe lá $50 \mathrm{~km}$, este número está indicando uma medida [...]. Ou número para ordenar; ou número como um código. Então, tem uma diferença aí do número para medir. Você encontra isso... os valores em reais, por exemplo, eles indicam uma medida (Rúbia, professora dos anos iniciais do ensino fundamental). 
A discussão elaborada por Rúbia exemplifica uma das muitas conexões existentes entre os diferentes conteúdos e temas da matemática - aqui envolvendo números e sentido de número e medidas (o que é medir) - que são, na BNCC (BRASIL, 2018), dois dos seus temas integrantes (KSM conexões transversais, auxiliares, de simplificação e de complexificação). 0 conhecimento da professora relativamente às formas como se pode entender o número (quantidade, medida, ordenação e código) associado à definição (e sentido) de número, compõe parte do conteúdo do KoT definições e fundamentos. Por outro lado, há que notar que um conhecimento relativo aos diferentes significados/sentidos de número não corresponde, necessariamente, a um conhecimento associado às diferenças específicas entre o significado de cada um desses sentidos (KoT - propriedades; registros de representação; fenomenologia e aplicações).

\begin{abstract}
Mas, por exemplo, se você tem $1 \mathrm{~kg}$ de carne, você também tá... para você saber... É uma quantidade, mas você sempre compara com o padrão... Na balança, você tem, né? (Amanda, psicopedagoga).

É uma quantidade que compara com um padrão, não é uma quantidade cardinal, não é uma quantidade que você conta em unidades (Rúbia, professora dos anos iniciais do ensino fundamental).
\end{abstract}

É diferente para quantidade (Amanda, psicopedagoga).

É diferente em termos de função, ela tem a função de indicar uma medida (Rúbia, professora dos anos iniciais do ensino fundamental).

A distinção efetuada por Rúbia entre os sentidos de número, identificando dois tipos (número como cardinal e número como medida), evidencia, mais uma vez, as conexões existentes entre os sentidos do número e as medidas, pois identificar a quantidade de vezes que a unidade de referência está contida no todo a ser medido ou que parte dessa unidade deverá ser considerada, associa-se, por um lado, a compreender a noção de cardinalidade do número (CEBOLA, 2002). Por outro lado, essas conexões sustentam um conhecimento que se vincula a compreender que atribuir um valor numérico - quantidade, portanto - corresponde a um dos princípios da atividade de medir (CLEMENTS; STEPHAN, 2004).

O estabelecimento dessas conexões sustenta-se no conhecimento do professor em relação à diferença entre aplicação (quando se usa o número como quantidade) e representação (número representativo de uma medida), conteúdos esses correspondentes à dimensão do KoT (sentido de número; 
DI BERNARDO, Rosa; POLICASTRO, Milena Soldá; ALMEIDA, Alessandra Rodrigues de; RIBEIRO, Miguel; MELO, Juscier Mamoré de; AIUB, Mariana

propriedades do número; procedimentos e fundamentos para medir; fenomenologia e aplicações do número em relação à medida) e do KSM (conexões auxiliares - o sentido de número atribuindo significado à medida).

Outra evidência sobre os conteúdos do conhecimento do professor, relacionados, particularmente, com as noções de definição de grandeza e de unidade de medida, está patente na argumentação de Wilson, um dos participantes (aqui apresentado em três trechos, por motivos operacionais): “Eu acho que a criança, como ela não tem ainda a noção de grandeza, as grandezas e a noção de unidade de medida, ela vai muito por comparação mesmo. Ela quantifica e compara: você tem três balas, ela tem cinco. 'Ah, então ela tem mais"”.

Wilson revela um conhecimento associado à necessidade de reconhecer a relação entre a unidade de medida e a grandeza que se está medindo, para que, apoiado pela noção de número enquanto cardinal (quantidade), seja possível referir-se a uma medida. Ilustra assim a ideia de que o número isolado (encarado como quantidade) possui um significado distinto do número como cardinal associado a uma unidade de medida (num processo de comparação com outra quantidade). De fato, quando aquilo que se está comparando não representa uma grandeza, o processo de comparação não está relacionado com a atividade de medir (que corresponde ao KoT - fundamentos; procedimentos). No exemplo do professor, ao considerar as "balas" como tendo a mesma forma, volume, massa etc. (balas idênticas - já que não se pode repetir a mesma bala), pode-se considerar que esses elementos não representam uma grandeza; logo, a comparação efetuada se dá entre o cardinal de dois conjuntos em que os elementos são balas ("você tem três balas, ela tem cinco. 'Ah, então ela tem mais"'), a partir de uma correspondência um a um. Porém, isso não se configura como uma comparação no sentido da atividade de medir, pois não se está efetuando uma "relação da medida com um valor numérico" (CLEMENTS; STEPHAN, 2004). Por outro lado, se as balas tiverem, por exemplo, formas, volume ou massas diferentes, pode-se considerar que uma é submúltiplo da outra e, nesse sentido, para identificar qual dos dois grupos "tem mais", é necessário que se estabeleça uma unidade comum de comparação, com a qual se possam medir, ao mesmo tempo, os dois conjuntos, o que se configura, efetivamente, como um processo de medição (CLEMENTS; STEPHAN, 2004).

No entanto, quando for possível identificar mais de uma grandeza a ser comparada, como, por exemplo, moeda (considerada como múltiplo e submúltiplo da mesma grandeza, por possuir formas distintas) e valor 
monetário, devem ser devidamente identificadas as grandezas a serem medidas, as unidades correspondentes à medição de cada uma delas, além de clarificar a impossibilidade de comparar grandezas de naturezas distintas (KoT - definições e fundamentos; KPM - formas de validação de um argumento).

Wilson assim argumenta: “Aí entra na questão do dinheiro, você tem 20 moedas de 5 centavos, eu tenho uma moeda de 1 real, a criança vai falar que quem tem a maior quantidade, para ela, é quem tem o maior valor”. Embora Wilson não tenha deixado claro (é possível, inclusive, que não seja consciente disso), seu comentário evidencia um reconhecimento de que as moedas, por serem de tipos diversos e formas diferentes (embora, verbalmente, sejam identificadas pela mesma palavra), representam a mesma grandeza, e um dos tipos é submúltiplo do outro (a moeda de 5 centavos, por exemplo, é submúltiplo da moeda de 1 real). Assim, este exemplo é distinto do anterior, associado às balas - pois, neste último, não há distinção entre os tipos de moedas, como ocorria com os "tipos" de balas. Essa forma de modelar uma situação real, buscando tornar compreensíveis os procedimentos e os raciocínios relacionados com a atividade de medir, bem como um conhecimento da linguagem e do papel dos símbolos, associa-se ao conhecimento especializado do professor relativo à prática matemática (KPM - uso da linguagem e dos símbolos e formas de validação de um argumento).

O conteúdo do conhecimento do professor, associado aos "comos", aos "porquês" e às características de um resultado (com uma ausência de elementos referentes aos tipos de conexões de simplificação e complexificação [KSM] e aos procedimentos relacionados com a medida: o que medir, como medir e quando medir [KoT], além dos fundamentos que sustentam a atividade de medir - unidade, grandeza e quantidade - [KoT - procedimentos e fundamentos]), sustenta sua argumentação nas exemplificações que fornece para distinguir a situação que parece não corresponder a uma medida (o caso das balas) daquela em que são evidentes os fundamentos da atividade de medir (o caso das moedas). É de salientar ainda um conhecimento associado a uma conexão entre dois campos do conhecimento matemático - o campo numérico (quantidade) e o sistema monetário -: grandeza (KoT - fenomenologia e aplicações no âmbito das medidas). Esse intercâmbio evidencia as potencialidades dessas conexões para desenvolver o conhecimento matemático, relacionado, por exemplo, com a noção de igualdade - equivalência e sentidos do símbolo "=" (TRIVILIN; RIBEIRO, 2015) - (KSM - conexões auxiliares e transversais). 
Ao continuar a sua argumentação relativamente à diferença entre quantidade e valor, no caso do contexto monetário, diz Wilson: "Eu tenho uma nota de 100 e cinco notas de 10, ela vai achar que a maior quantidade é quem tem cinco notas, então a criança, eu acredito, que ela vai por comparação de quantidades, não tem ainda essa noção do valor das grandezas e das unidades de medida".

A relação entre unidade de medida e quantidade permite que, no contexto de distintas grandezas (notas e valor monetário), seja possível explicitar a relação de equivalência ou desigualdade de quantidades, mesmo quando os símbolos associados a elas forem diferentes, desde que representem quantidades múltiplas ou submúltiplas (KoT - tipos de representação e propriedades; KSM - conexões de simplificação/complexificação ou auxiliares). No caso das moedas, a representação escrita das quantidades na forma decimal (uma moeda de $R \$ 1,00$ e 20 moedas de $R \$ 0,05$ ) possibilita que, por um lado, identifique-se o real como a unidade de referência (e os centavos, um submúltiplo deste) e estabeleça-se a comparação das quantidades em termos de valor monetário. Por outro lado, a verbalização associada a essa escrita, se considerada isoladamente da grafia, não denota que a unidade de referência seja o real. Poderá, portanto, contribuir para um equívoco, pois se trata de uma grandeza (o real) sendo comparada em duas de suas formas - múltiplo e submúltiplo (KoT - tipos de representação e formas de verbalização associada e KSM - conexões auxiliares). É importante ressaltar que ao professor cumpre, também, um tipo de conhecimento que lhe permita navegar entre as distintas formas de representação de um mesmo objeto matemático (RIBEIRO, 2011), ao mesmo tempo que relaciona cada uma dessas representações a um tipo de linguagem (POLICASTRO; ALMEIDA; RIBEIRO, 2018) que corresponda ao efetivamente representado, de modo a permitir que os alunos entendam o que estão fazendo, por que o estão fazendo e quando podem fazer (no sentido de que possam generalizar procedimentos e abstrair raciocínios).

Analisando o conhecimento especializado do professor relativamente ao tema de medidas, em uma tarefa com foco em "o que é medir?", em termos investigativos, evidenciou-se a necessidade de desvincular-se do foco específico da questão, dando "um passo atrás”, para especificar/detalhar as conexões existentes entre os três pilares que fundamentam a atividade de medir (quantidade - unidade - grandeza). Tomando esses pilares dois a dois, e com o intuito de melhor caracterizar as conexões entre eles e as implicações dessas conexões, tanto do ponto de vista da pesquisa quanto da 
formação - o que, necessariamente, na perspectiva que assumimos, busca resultados que melhorem a prática dos professores e das aprendizagens dos alunos -, apresentamos, na Figura 2, um esquema que procura não somente explicitar essas conexões, mas também evidenciar de que forma tais conexões fornecem elementos que sustentam a noção transversal de "comparação" que fundamenta a atividade de medir.

Figura 2 - Esquema das conexões entre unidade - grandeza - quantidade

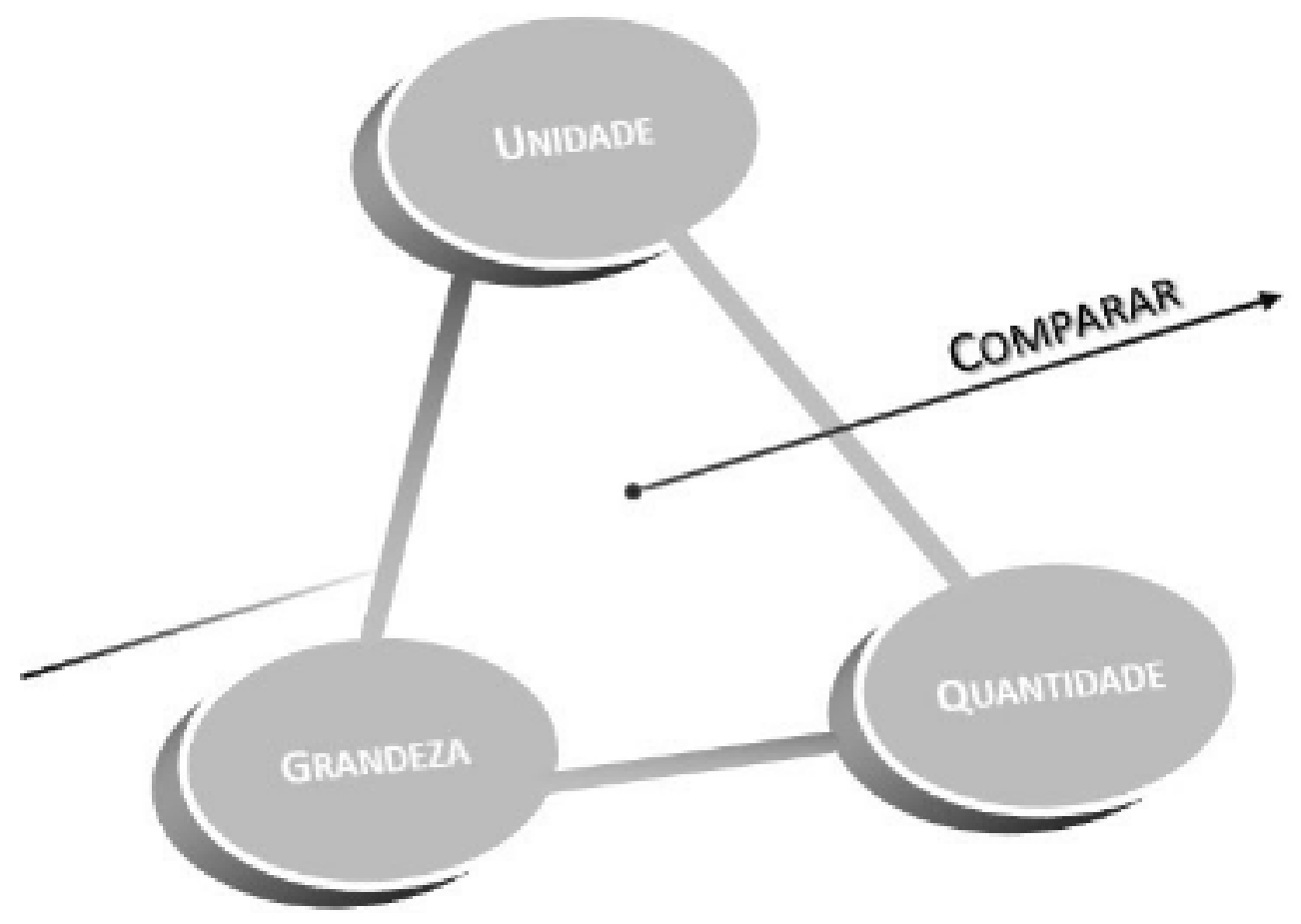

Fonte: Elaborada pelos autores (2018).

É essencial que as noções de grandeza e quantidade estejam consolidadas e seus sentidos estejam intrinsecamente relacionados, para que a introdução de um símbolo que represente a noção de grandeza (reais ou $\mathrm{R} \$$, por exemplo) não represente meramente uma "marca" que acompanha o número (CEBOLA, 2002). De fato, a relação entre o valor numérico associado a uma quantidade e a grandeza que a ele está vinculada deverá contribuir para a abstração da ideia de medida (sustentando e sendo sustentada pela noção de comparação), entre outros aspectos relacionados com noções transversais à matemática (como, por exemplo, a noção de proporcionalidade).

Por outro lado, as conexões entre unidade e quantidade poderão, por exemplo, implicar/contribuir no/para o desenvolvimento do raciocínio transitivo, associado à construção da noção de comparação entre quantidades (se uma 
nota de 50 reais corresponde a uma quantidade menor que uma nota de 100 reais, logo, dez notas de 5 reais correspondem a uma quantidade menor que uma nota de 100 reais), o que, necessariamente, dependerá da consolidação da noção de equivalência (e do papel do símbolo “=”).

Já a noção de equivalência (e o papel do símbolo “=”) poderá ser desenvolvida, por exemplo, a partir das conexões que se podem estabelecer entre unidade e grandeza, pois, quando se comparam medidas expressas por uma mesma grandeza, envolvendo múltiplos e submúltiplos dessa grandeza, é preciso que se identifique qual a unidade de comparação para que uma possível equivalência, por exemplo, seja compreendida (1 kg = $1.000 \mathrm{~g})$.

\section{CONSIDERAÇÕES FINAIS}

Embora nesta investigação tenhamos focado essencialmente os três subdomínios do MK do professor, uma vez que a tarefa estava intencionalmente desenhada para aceder e desenvolver o MTSK dos professores relativamente a tais subdomínios, os professores exteriorizaram também aspectos relativos ao PCK. Tal fato contribui para que se possa, por um lado, melhor compreender o duplo papel do modelo: como ferramenta analítica - que contribui para efetivamente analisar e discutir o conhecimento especializado do professor de/que ensina matemática - e como lente teórica, na qual todos os seis subdomínios (e as dimensões do conhecimento do professor a eles associadas) estão inter-relacionados e se alimentam mutuamente (MK e PCK sustentam a prática do professor e ambos influenciam e são influenciados pelas crenças do professor). Por outro lado, essa emergência dos comentários associados ao PCK, mesmo em um contexto desenhado para discutir o MK, revela também a necessidade de tarefas intencionais para aceder e desenvolver o PCK do professor relativamente ao tema das medidas - não se limitando a aspectos de conhecimento pedagógico geral, como tem sido uma tendência verificada nas pesquisas efetuadas de 2001 a 2012, analisadas pelo metaestudo recente (consultar, por exemplo, FIORENTINI; PASSOS; LIMA, 2016). Este foco das tarefas para a formação, considerando as suas especificidades, além de contribuir para a formação, deverá ampliar o entendimento sobre cada um dos subdomínios do MTSK do professor de/que ensina matemática, no que diz respeito, em particular, ao tema de medidas.

A pesquisa mostra que, em relação às medidas, um conhecimento especializado do professor refere-se às conexões existentes entre os três pilares - noções de quantidade, unidade e grandeza -, que fundamentam a medida e a 
atividade de medir. Assim, e por ser a medida um dos temas problemáticos para alunos e professores, torna-se essencial um aprofundamento da natureza, do conteúdo e dos tipos dessas conexões - assumindo aqui a necessidade de um lugar de destaque para a pesquisa com foco na prática matemática do professor e a elaboração de tarefas que busquem promover o desenvolvimento desse conhecimento associado a tais dimensões e conexões. Uma das evidências da pesquisa mostra a conexão entre os pilares quantidade e grandeza, que sustenta o desenvolvimento do conhecimento matemático relacionado com a noção - transversal à matemática - de igualdade (equivalência e sentido do símbolo “=”), e que pode ser assumida como uma das grandes ideias a trabalhar na formação. 0 desenvolvimento desse conhecimento especializado do professor (KSM) sustentará as decisões didático-pedagógicas implementadas posteriormente, que poderão implicar em diferentes oportunidades de aprendizagens facultadas aos alunos - tanto desde o ponto de partida como de chegada, passando pelas discussões matemáticas elaboradas e possibilitadas.

Assim, um dos aspectos essenciais que se deve considerar e promover na formação de professores de/que ensinam matemática corresponde ao aprofundamento do entendimento do papel das conexões (KSM) na prática e para a prática, contribuindo efetivamente para que, com essa formação, novos caminhos e possibilidades formativas possam ser efetivados, no sentido de contribuir para a melhoria da prática matemática. 


\section{Specialized Teacher Knowledge of Mathematics in early childhood and elementary education: connections in measurement}

Abstract: In this paper, we focused on evidence of specialized knowledge (from the perspective of Mathematics Teachers' Specialized Knowledge) of early childhood and elementary teachers when discussing a task designed to develop such knowledge, regarding on how the notions of quantity, unity and magnitudes are connected. The results revealed that the participants associate measurement to a specific unit of comparison (standard), followed by an identification of a numerical value and a symbol to represent/indicate the quantity of what was measured. The connections between the notions of quantity, unit and magnitudes allowed detailing the implications of choosing to focus on each connection as a starting point and opened up investigation possibilities about assuming each connection as a focal point in terms of teacher education.

Keywords: Specialized knowledge, measure, teacher education, early childhood education. 


\section{Conocimiento matemático especializado de profesores de la educación infantil y los años iniciales: conexiones con medidas}

Resumen: En este trabajo buscamos evidencias del conocimiento especializado (desde la perspectiva del Mathematics Teachers' Specialized Knowledge) de los profesores de la educación infantil y de los años iniciales de la enseñanza básica en la discusión de una tarea conceptualizada para desarrollar este conocimiento con respecto al modo como las nociones de cantidad, unidad y grandeza se articulan. Los resultados mostraron que los participantes revelan un conocimiento asociando medida al establecimiento de una referencia de comparación, seguido de la indicación de un valor numérico y un símbolo para representar/indicar la cantidad y lo que se mide. Las conexiones entre las nociones de cantidad, unidad y grandeza permiten detallar las consecuencias de optar por el trabajo a partir de cada uno de los ejes de conexión y abren posibilidades investigativas sobre asumir cada uno de los ejes como foco, en términos de formación.

Palabras clave: Conocimiento especializado. Medidas. Formación de profesores. Años iniciales. 
DI BERNARDO, Rosa; POLICASTRO, Milena Soldá; ALMEIDA, Alessandra Rodrigues de; RIBEIRO, Miguel;

\section{REFERÊNCIAS}

ALSINA, Á. Contextos y propuestas para la enseñanza de la estadística y la probabilidad en Educación Infantil: un itinerario didáctico. Contexts and proposals for teaching statistics and probability in Early Childhood Education: a didactic itinerary. Épsilon, v. 34, n. 95, p. 25-48, 2017.

BALL, D.; HILL, H. H.; BASS, H. Knowing mathematics for teaching: who knows mathematics well enough to teach third grade, and how can we decide? American Educator, n. Fall, p. 14-46, 2005.

BALL, D.; THAMES, M. H.; PHELPS, G. Content knowledge for teaching: what makes it special? Journal of Teacher Education, v. 59, n. 5, p. 389-407, 2008.

BERTOLINO, J. Matemática significativa: sequência didática para aprendizagem de área e perímetro no ensino fundamental. Revista Científica on-line Tecnologia, Gestão e Humanismo, v. 8, n. 1, 2017.

BRASIL. Secretaria de Educação Fundamental. Parâmetros curriculares nacionais: terceiro e quarto ciclos do ensino fundamental. Brasília: Ministério da Educação/Secretaria de Educação Média e Tecnológica, 1997. v. 1.

. Base Nacional Comum Curricular. Quarta edição. Brasília: Ministério da Educação, 2018.

CARAÇA, B. DE J. Conceitos fundamentais da matemática. Lisboa: Bertrand, 1963.

CARRILLO, J. et al. Determining specialised knowledge for mathematics teaching. In: CONGRESS OF EUROPEAN RESEARCH IN MATHEMATICS EDUCATION, 8., 2013, Antalya. Anais.... Antaya, Turquia: Erme, 2013.

CEBOLA, G. Do número ao sentido do número. Atividades de investigação na aprendizagem da matemática e na formação de professores. Lisboa: Secção de Educação e Matemática da Sociedade Portuguesa de Ciências da Educação, 2002.

CLEMENTS, D. H.; STEPHAN, M. Measurement in pre-K to grade 2 mathematics. In: CLEMENTS, J. S.; DI BIASE, A-M. Engaging young children in Mathematics: Standards for early childhood mathematics education. New Jersey: LEA, D.H., 2004. p. 299-317.

FIORENTINI, D.; PASSOS, C. L.; LIMA, R. C. L. Mapeamento da pesquisa 
acadêmica brasileira sobre o professor que ensina matemática: período 20012012. Campinas: FE/Unicamp, 2016.

FLORES-MEDRANO, E. et al. Nuestra modelación del conocimiento especializado del profesor de matemáticas, el MTSK. In: Un marco teórico para el conocimiento especializado del profesor de matemáticas. [s.l., s.n.], 2013.

GROSSMAN, P. L. Learning to practice: the design of clinical experience in teacher preparation. Policy Brief, Washington. D.C.: NEA, maio 2010.

HEUVEL-PANHUIZEN, M. V. DEN; ELIA, I. Kindergartners' performance in length measurement and the effect of picture book reading. ZDM, v. 43, n. 5, p. 621$635,1^{\circ}$ out. 2011.

HIEBERT, J.; GROUWS, D. The effects of classroom mathematics teaching on students' learning. In: LESTER, F. (Ed.). Handbook of research on mathematics teaching and learning. Nova York: Information Age Publishing, 2007. p. 371404.

HILL, H. C.; ROWAN, B.; BALL, D. Effects of teachers' mathematics knowledge for teaching on student achievement. American Education Research Journal, v. 42, n. 2, p. 371-406, 2005.

ITÁLIA. Indicazioni nazionali per il curricolo della scuola dell'infanzia e del primo ciclo d’instruzione. Itália: Ministério da Educação, 2012.

MUIR, T. What is a reasonable answer? Ways for students to investigate and develop their number sense. Australian Primary Mathematics Classroom, v. 17, n. 1, p. 21-28, 2012.

NATIONAL GOVERNORS ASSOCIATION CENTER FOR BEST PRACTICES; COUNCIL OF CHIEF STATE SCHOOL OFFICERS. Common Core State Standards Initiative. [s.l.] National Governors Association Center for Best Practices, 2010.

NORWEGIAN. Framework plan for kindergartens. Nynorsk and Sami: Directorate for Education and Training, 2017.

NYE, B.; KONSTANTOPOULOS, S.; HEDGES, L. How large are teacher effects?. Educational evaluation and policy analysis. Educational Evaluation and Policy Analysis, v. 26, n. 3, p. 237-257, 2004.

POLICASTRO, M. S.; ALMEIDA, A. R.; RIBEIRO, M. Conhecimento especializado 
DI BERNARDO, Rosa; POLICASTRO, Milena Soldá; ALMEIDA, Alessandra Rodrigues de; RIBEIRO, Miguel;

revelado por professores da educação infantil e dos anos iniciais no tema de medida de comprimento e sua estimativa. Revista Espaço Plural, (no prelo), 2018.

PORTUGAL. Programa e metas curriculares - Matemática. [s.l.] Ministério da Educação e Ciência, 2013.

RIBEIRO, M. Abordagem aos números decimais e suas operações: a importância de uma "eficaz navegação" entre representações. Educação e Pesquisa, v. 37, n. 2, p. 407-422, 2011.

- Tarefas para alunos e tarefas de formação: discutir o conhecimento especializado do professor e do professor de professores de matemática. In: JORNADAS NACIONALES DE EDUCACIÓN MATEMÁTICA - SOCHIEM, 20., 2106, Valparaíso. Anais... Valparaíso: Sochiem, Instituto de Matemática de la Pontificia Universidad Católica de Valparaíso - Chile, 2016.

. Das generalidades às especificidades do conhecimento do professor que ensina matemática: metodologias na conceitualização (entender e desenvolver) do conhecimento interpretativo. In: REUNIÃO NACIONAL DA ANPED, 38., 2017, São Luís. Anais... [s.l.] Sbem-SP, 2018.

; CARRILLO, J. Knowing mathematics as a teacher. In: CONGRESS OF EUROPEAN SOCIETY FOR RESEARCH IN MATHEMATICS EDUCATION, 7., 2011, Rzeszów. Anais... Rzeszów, Polônia: Erme, 2011.

RIBEIRO, M. et al. Mathematics teacher knowledge, beliefs, and identity: some reflections on the current state of the art. In: KRAINER, K.; VONDROVÁ, N. (Ed.) CONGRESS OF THE EUROPEAN SOCIETY FOR RESEARCH IN MATHEMATICS EDUCATION, 9., 2016. Anais... Praga, República Tcheca, 2016.

- Intertwining noticing and knowledge in video analysis of self practice: the case of Carla. In: CONGRESS OF THE EUROPEAN SOCIETY FOR RESEARCH IN MATHEMATICS EDUCATION, 10. 2017, Dublin. Anais... Dublin, 2017.

RIBEIRO, M.; MELLONE, M.; JAKOBSEN, A. Interpreting students' non standard reasoning: insights for mathematics teacher education practices. For the Learning of Mathematics, v. 36, p. 8-13, 2016.

ROWLAND, T. et al. Developing primary mathematics teaching: Reflecting on practice with the knowledge quartet. [s.l.] Sage, 2009.

SHULMAN, L. Those who understand: knowledge growth in teaching. Educational Researcher, v. 15, n. 2, p. 4-14, 1986. 
SILVA, I. L. D. et al. Orientações curriculares para a educação pré-escolar. [s.l.] Brasília: Ministério da Educação/Direção-Geral da Educação (DGE), 2016.

SILVA, J. A. da; BELLEMAIN, P. B.; BORBA, R. E. de S. R. Análise de itens da Provinha Brasil de Matemática referentes a Grandezas e Medidas. Perspectivas da Educação Matemática, v. 9, n. 21, 28 dez. 2016.

TARDIF, M. Saberes docentes e formação profissional. 15. ed. Petrópolis: Vozes, 2002.

TRIVILIN, L.; RIBEIRO, A. J. Conhecimento matemático para o ensino de diferentes significados do sinal de igualdade: um estudo desenvolvido com professores dos Anos Iniciais do Ensino Fundamental. Bolema: Boletim de Educação Matemática, Rio Claro, v. 29, n. 51, p. 38-59, 2015.

WATTS, T. W. et al. What is the long-run impact of learning mathematics during preschool? Child Development, v. 89, n. 2, p. 539-555, 1으 mar. 2018.

\section{AgRADECIMENTOS}

Este texto foi produzido tomando por base o trabalho desenvolvido no âmbito do projeto "Conhecimento matemático especializado do professor que ensina matemática na educação infantil e nos anos iniciais: um foco em conteúdos de Geometria", processo número 2016/22557-5, Fundação de Amparo à Pesquisa do Estado de São Paulo (Fapesp).

\section{SOBRE OS AUTORES}

Rosa Di Bernardo é mestre em Scienze della Formazione Primaria pela Università Suor Orsola Benincasa (Unisob - Napoli, Itália). Atualmente é doutoranda do Programa de Pós-Graduação Multiunidades em Ensino de Ciências e Matemática da Educação Matemática (Pecim) pela Universidade Estadual de Campinas (Unicamp).

E-mail: rosadibernardo92@gmail.com.

Milena Soldá Policastro é mestre em Ensino de Ciências e Matemática pela Universidade de São Paulo (USP). Atualmente é doutoranda do Programa de Pós-Graduação da Faculdade de Educação da Unicamp. Atua em formação de professores e é coordenadora de matemática dos anos iniciais na rede privada de ensino básico.

E-mail:mitapolicastro@gmail.com. 
Alessandra Rodrigues de Almeida é doutora pelo Programa de Pós-Graduação Multiunidades em Ensino de Ciências e Matemática (Pecim) da Unicamp. Atualmente é professora da Faculdade de Educação da Pontifícia Universidade Católica de Campinas (PUC-Campinas).

E-mail: alessandraalmeida628@gmail.com

Miguel Ribeiro é doutor em educação matemática pela Universidade de Huelva (Espanha). Atualmente é professor titular da Faculdade de Educação da Unicamp.

E-mail:cmribas78@gmail.com

Juscier Albertino Mamoré de Melo é licenciado em Matemática pela Universidade Estadual de Campinas (Unicamp). Atualmente é mestrando no Pecim-Unicamp.

E-mail: jjmamore@gmail.com.

Mariana Aiub é licenciada em Matemática pela Universidade Estadual de Campinas (Unicamp). Atualmente é mestranda pelo Programa de PósGraduação Multiunidades em Ensino de Ciências e Matemática (Pecim/ Unicamp).

E-mail: marianamra@gmail.com

Recebido em: maio de 2018

Aprovado em: junho de 2018 